\title{
FAMILIES OF MODULAR EIGENFORMS
}

\author{
F. GOUVÊA AND B. MAZUR
}

\begin{abstract}
This article is an expansion of the notes to a one-hour lecture for an MSRI workshop on computational number theory. The editors of Mathematics of Computation kindly asked us to submit these notes for publication, and we are enormously pleased to do so. Our original audience did not consist of experts in the field of modular forms, and we have tried to keep this article accessible to nonexperts.
\end{abstract}

We have made an experimental investigation of certain arithmetic conjectures using MACSYMA. This investigation requires a search for certain modular eigenforms of high weight. These computations pose problems which we feel may be interesting on their own. We are novices here, and we seek advice from people more experienced in making computations of an analogous sort. We are, in fact, deeply grateful to J. F. Mestre, who came to our aid and who vastly extended our computations using PARI. ${ }^{1}$ Mestre has graciously allowed us to present his computations in this article.

The kind of families we have in mind, as in the title of this lecture, has as its prototypical example the standard family of classical Eisenstein series of level 1 and weight $k$ for $k=4,6,8, \ldots$, whose Fourier expansions are given by

$$
G_{k}(z)=-b_{k} / 2 k+\sum_{n=1}^{\infty} \sigma_{k-1}(n) \cdot q^{n}
$$

with $q=e^{2 \pi i z}, \sigma_{k-1}(n)=$ the sum of the $(k-1)$ st powers of all positive divisors of $n$, and $b_{k}$ the $k$ th Bernoulli number, so that the constant term of the Fourier expansion of $G_{k}$ is also given as

$$
-b_{k} / 2 k=(-2 \pi)^{-k} \cdot \Gamma(k) \cdot \zeta(k),
$$

where $\Gamma(s)$ is the gamma function and $\zeta(s)$ the Riemann zeta function. These modular forms intuitively "fit into a family" in a number of senses. First, they are the unique series of level-1 noncuspidal eigenforms for the Hecke operators. But also, the Fourier series for $G_{k}(z)$ extend in a (multivalued) complex analytic manner for $k$ any complex number, if we interpret the constant term $-b_{k} / 2 k$ as the right-hand side of (2).

It might be tempting to ask whether there are other such families of classical eigenforms for the Hecke operators, cuspidal, say, of some level $N$, parametrized

Received September 24, 1990; revised November 30, 1990.

1991 Mathematics Subject Classification. Primary 32N99, 11 Y99.

${ }^{1}$ Developed by C. Batut, D. Bernardi, H. Cohen, and M. Olivier.

(C) 1992 American Mathematical Society $0025-5718 / 92 \$ 1.00+\$ .25$ per page 
by the weight $k$, and which fit so neatly into a "complex analytic" family of Fourier series. We would not be surprised if the answer to this were: no. (The existence of the above "analytic" family of Eisenstein series is related to the existence of a continuous spectrum for the spectral decomposition of the $G_{\mathrm{A}^{-}}$representation space $L^{2}\left(G_{\mathbf{A}} / G_{\mathbb{Q}}\right)$, where $G=\mathrm{SL}_{2}$.)

Nevertheless, if we replace the phrase "complex analytic family" by " $p$-adic analytic family," the situation changes completely. This is a story begun by Kummer, and continued in various directions by Iwasawa, Leopold-Kubota, Serre, Swinnerton-Dyer, Katz, Deligne-Ribet, Hida, and others.

\section{The EISENSTEIN FAMILY}

To illustrate what we might mean by a " $p$-adic family" of eigenforms for the Hecke operators, consider, for $p$ a prime number, the following slightly modified version of the family (1) of Eisenstein series above (studied in depth in [8]):

$$
G_{k}^{*}(z)=-\left(1-p^{k-1}\right) b_{k} / 2 k+\sum_{n=1}^{\infty} \sigma_{k-1}^{*}(n) \cdot q^{n},
$$

where $k$ is supposed not congruent to $0 \bmod p-1$. (This last condition excludes $p=2$ and 3.)

Here, $\sigma_{k-1}^{*}(n)$ is the sum of the $(k-1)$ st powers of all positive divisors of $n$ which are relatively prime to $p$.

It is evident from Fermat's little theorem that

$$
\sigma_{k^{\prime}-1}^{*} \equiv \sigma_{k-1}^{*} \quad \text { modulo } p^{n+1},
$$

provided $k^{\prime} \equiv k$ modulo $(p-1) p^{n}$. Classical formulae (closely related to what are known as the Kummer congruences) guarantee congruences analogous to (3) for the constant terms of the Fourier expansion of $G_{k}(z)$ and $G_{k^{\prime}}(z)$. In other words, we have that

$$
G_{k} \equiv G_{k^{\prime}} \quad \text { modulo } p^{n+1},
$$

provided $k^{\prime} \equiv k$ modulo $(p-1) p^{n}$. Here, two power series with rational coefficients in the variable $q$ are said to be congruent $\bmod m$ ("三 $\bmod m$ ") if their difference has the property that its coefficients, when expressed as fractions in lowest terms, have their numerators divisible by $m$.

\section{Cuspidal Classical EIGENFORMS}

Let us take then the " $p$-adic continuity" phenomenon given in (4) as a prototype and seek similar families among cuspidal eigenforms. We shall work exclusively with classical cuspidal modular forms $f$ (of weight $k \geq 1$ ) for congruence groups of the form $\Gamma_{0}(N p)$, where $N$ is an integer prime to $p$ (the "tame" level). The Hecke operators $T_{l}$ for $l$ not dividing $N \cdot p$, and the Atkin operators $U_{l}$ for prime numbers $l$ dividing $N \cdot p$, act on, and preserve, the space of such modular forms, their effect on Fourier expansions being given by the following formulae:

If $f=\sum_{n=1}^{\infty} a_{n} q^{n}$ is such a modular form of weight $k$, then

$$
T_{l}(f)=\sum a_{l n} q^{n}+l^{k-1} \sum a_{n} q^{l n}
$$


and

$$
U_{l}(f)=\sum a_{\ln } q^{n}
$$

By a classical eigenform (for short) we shall mean a cuspidal modular form (of weight $k$ for some integer $k \geq 0$, of tame level $N$ for some positive integer $N$ prime to $p$ ) which is an eigenvector for the Hecke operators $T_{l}$ for $l$ not dividing $N \cdot p$ and for the Atkin operators $U_{l}$ for $l$ dividing $N \cdot p$, and such that $f$ has its first Fourier coefficient equal to 1 . It then follows that all the Fourier coefficients of $f$ are algebraic integers which generate a field $K_{f}$ of finite degree over $\mathbb{Q}$.

\section{EIGENFORMS (WITH COEFFICIENTS IN $\mathbb{C}_{p}$ )}

Let $\mathbb{C}_{p}$ denote the completion of an algebraic closure of $\mathbb{Q}_{p}$. This is an amply large field (abstractly isomorphic to the field of complex numbers) in which all our coefficients will lie, and which is "set up" to deal with congruences modulo powers of $p$. We imagine ${ }^{2}$ that we have fixed an isomorphism between $\mathbb{C}$ and $\mathbb{C}_{p}$ and then, given a classical eigenform $f$ as above, we transport the Fourier coefficients of $f$ to $\mathbb{C}_{p}$ via this isomorphism. In this way we consider the Fourier expansion of $f$ as lying in the ring of power series $\mathbb{C}_{p}[[q]]$ and refer to this Fourier expansion as an eigenform with coefficients in $\mathbb{C}_{p}$, or (when the prime $p$ is understood) as an eigenform. We have avoided using the phrase " $p$-adic modular form" because this has been used in the literature (cf. $[8,7,3])$ to refer to various kinds of $p$-adic completions of the space of classical modular forms.

\section{The SLOPE OF AN EIGENFORM (WITH COEFFICIENTS IN $\mathbb{C}_{p}$ )}

Recall that we have fixed the prime number $p$, and an integer $N$ relatively prime to $p$.

If $f$ is an eigenform, define $\alpha(f)$, the slope of $f$, to be the $p$-adic ord of its $U_{p}$-eigenvalue, i.e.,

$$
\alpha(f)=\operatorname{ord}_{p}\left(a_{p}(f)\right) \text {, }
$$

where we normalize ord $\operatorname{ord}_{p}$ by requiring that $\operatorname{ord}_{p}(p)=1$.

Let $L$ be an extension field of $\mathbb{Q}_{p}$ contained in $\mathbb{C}_{p}$. Let $S(k ; L)$ be the (finite-dimensional) $L$-vector space of all cuspforms with Fourier coefficients in $L$ which are of level $N p$ and weight $k$. The Atkin operator $U_{p}$ acts on $S(k ; L)$, and we have a direct sum decomposition

$$
S(k ; L)=\bigoplus S(k ; L)^{\alpha}
$$

$(\alpha \in \mathbb{Q} ; \alpha \geq 0)$, where $S(k ; L)^{\alpha}$ is the slope $\alpha$ eigensubspace of $S(k ; L)$. This subspace can be defined as the image of $P_{\alpha}\left(U_{p}\right)$ acting on $S(k ; L)$, where $P_{\alpha}(T)$ is the factor of the characteristic polynomial of the operator $U_{p}$ on $S(k ; L)$ which contains all eigenvalues of $U_{p}$ whose $\operatorname{ord}_{p}$ is different from $\alpha$. Note that if $L=\mathbb{C}_{p}$, then $S(k ; L)^{\alpha}$ is the sum of all the generalized eigenspaces for the operator $U_{p}$ on $S(k ; L)$ whose eigenvalues have $p$-adic ord equal to $\alpha$. We refer to the decomposition of $S(k ; L)$ into the $S(k ; L)^{\alpha}$ as the slope decomposition of $S(k ; L)$. Note that

$$
S(k ; L)^{\alpha} \cong S\left(k ; \mathbb{Q}_{p}\right)^{\alpha} \otimes L .
$$

\footnotetext{
${ }^{2}$ We can, in fact, hardly imagine doing this.
} 
Write $S(k)=S\left(k ; \mathbb{C}_{p}\right)$ and $S(k)^{\alpha}=S\left(k ; \mathbb{C}_{p}\right)^{\alpha}$, for short.

One can get, by elementary means, some information on the slopes that can occur in each weight $k$. First of all, divide the eigenforms of weight $k$ into two classes: " $p$-oldforms" and " $p$-newforms"; these are defined as follows.

"p-oldforms": Consider a classical (normalized) cuspform

$$
\varphi=\sum_{n=1}^{\infty} A_{n} q^{n} \quad\left(A_{1}=1\right)
$$

of weight $k$ on $\Gamma_{0}(N)$, with Fourier coefficients in $\mathbb{C}_{p}$, which is an eigenform for the Hecke operators $T_{l}$ for $l$ not dividing $N$ and for the operators $U_{l}$ for $l$ dividing $N$. Since $p$ does not divide $N, \varphi$ is an eigenform for the $T_{p}$-operator, so that $T_{p}(\varphi)=A_{p} \varphi$. The form $\varphi$ of level $N$ gives rise to a two-dimensional space of modular forms of level $N p$ generated by the forms $\varphi$ and by

$$
V_{p} \varphi=\sum_{n=1}^{\infty} A_{n} q^{n p},
$$

this space being stable under the action of the Hecke operators $T_{l}$ for $l$ not dividing $N p$ and by the Atkin operators $U_{l}$ for $l$ dividing $N p$. We may diagonalize the action of $U_{p}$ on this two-dimensional space to get two eigenforms. Specifically, let $\rho^{\prime}$ and $\rho^{\prime \prime}$ be the roots of the polynomial

$$
X^{2}-A_{p} X+p^{k-1} \text {. }
$$

Put

$$
\varphi_{p}^{\prime}:=\left(1-\rho^{\prime \prime} V_{p}\right)(\varphi) \text { and } \varphi_{p}^{\prime \prime}:=\left(1-\rho^{\prime} V_{p}\right)(\varphi) \text {. }
$$

Then $\varphi_{p}^{\prime}$ and $\varphi_{p}^{\prime \prime}$ are both eigenforms on $\Gamma_{0}(N p)$, with $U_{p}$-eigenvalues $\rho^{\prime}$ and $\rho^{\prime \prime}$, respectively. They are distinct if and only if $\rho^{\prime}$ and $\rho^{\prime \prime}$ are distinct.

Let us call such a pair of eigenforms, coming from the same newform, twin ( $p$-old) eigenforms. The terminology is justified in that, for example, all the eigenvalues of the Hecke operators $T_{l}(l$ not dividing $N \cdot p)$ and $U_{l}(l$ dividing $N$ ) agree on $\varphi, \varphi_{p}^{\prime}$, and $\varphi_{p}^{\prime \prime}$. The associated Galois representations of $\varphi_{p}^{\prime}$ and $\varphi_{p}^{\prime \prime}$ are equal; these Galois representations come, of course, from the same automorphic representation.

If $\alpha^{\prime}=\operatorname{ord}_{p}\left(\rho^{\prime}\right)$ and $\alpha^{\prime \prime}=\operatorname{ord}_{p}\left(\rho^{\prime \prime}\right)$, then $\varphi_{p}^{\prime}$ and $\varphi_{p}^{\prime \prime}$ have slopes $\alpha^{\prime}$ and $\alpha^{\prime \prime}$, respectively. Since $\rho^{\prime} \cdot \rho^{\prime \prime}=p^{k-1}$, these slopes add to $k-1$. We will always order $\rho^{\prime}$ and $\rho^{\prime \prime}$ so that $\alpha^{\prime} \leq \alpha^{\prime \prime}$.

To summarize, $p$-oldforms of weight $k$ have slopes between 0 and $k-1$, and (except for the rare case when $\rho^{\prime}=\rho^{\prime \prime}$ ) come in pairs ("twins") whose slopes add up to $k-1$.

" $p$-newforms": There is a trace function $\operatorname{Tr}$ that maps forms of level $N p$ to forms of level $N$. The simplest way to define $p$-newforms is to say that they are those forms of level $N p$ whose trace to level $N$ is zero. As in [1], this definition allows one to obtain their slopes: if $w_{p}$ is the usual involution of $S(k)$ attached to the prime $p$, we have

$$
\operatorname{Tr}(f)=f+p^{1-k / 2} U_{p} w_{p}(f) .
$$

Thus, on the space of $p$-newforms, we have $U_{p}=p^{k / 2-1} w_{p}$. Now, if $f$ is a $p$-newform that is also an eigenform (for the Hecke operators $T_{l}$ for $l$ not 
dividing $N p$, and for the $U_{l}$ for $l$ dividing $N p$ ), it is an eigenform for $w_{p}$. Since $w_{p}$ is an involution, we have $w_{p}(f)= \pm f$. It follows that

$$
U_{p}(f)= \pm p^{k / 2-1} f,
$$

and so that the only slope that can occur in the "new" part of $S(k)$ is $\alpha=k / 2-1$. We will often use this face to conclude that some eigenform is actually a $p$-oldform, i.e., comes from level $N$, and therefore has a twin.

To summarize, the only possible slopes $\alpha$ for which $S(k)^{\alpha}$ is nonzero lie in the range $\alpha \in \mathbb{Q}, 0 \leq \alpha \leq k-1$. Since the slope decomposition is in fact defined over $\mathbb{Q}_{p}$, we also have that the dimension of $S(k)^{\alpha}$ is divisible by the denominator of the rational number $\alpha$.

5. IS THE DIMENSION OF THE SLOPE $\alpha$ SUBSPACE "LOCALLY CONSTANT" AS A FUNCTION OF THE WEIGHT?

Fix a slope $\alpha$, and consider the function

$$
k \mapsto d(k, \alpha)=\operatorname{dim}_{\mathbb{C}_{p}} S(k)^{\alpha} .
$$

We conjecture that this function of $k$ is locally constant in an appropriate " $p$-adic topology." More specifically, and more precisely:

Conjecture 1. Fix a slope $\alpha$, and let $k_{1}$ and $k_{2}$ be integers satisfying

(1) both $k_{1}$ and $k_{2}$ are greater than or equal to $2 \alpha+2$;

(2) $k_{1} \equiv k_{2} \bmod p^{n}(p-1)$ for an integer $n \geq \alpha$.

Then $d\left(k_{1}, \alpha\right)=d\left(k_{2}, \alpha\right)$.

Conjecture 1 in the case of slope $\alpha=0$ is true for $p \geq 5$ (cf. [6,5]).

J. F. Mestre has accumulated some evidence for this conjecture, for tame level $N=1$, and for positive slope. For example:

$p=2$ : Here, Mestre has calculated $d(k, \alpha)$ for all $k \leq 256$, and all $\alpha$. The data is consistent with the above conjecture, and indeed with a refinement of it:

(?) $d\left(k_{1}, \alpha\right)=d\left(k_{2}, \alpha\right)$ if $k_{1}$ and $k_{2}$ are greater than or equal to $2 \alpha+2$ and $k_{1} \equiv k_{2} \bmod 2^{n-1}$ for an integer $n \geq \alpha$.

Specifically, within the range $4 \leq k \leq 256$, one has

(a) $S(k)^{\alpha}=0$ for $\alpha=0,1$, and 2 (all $k$ );

(b) $d(k, 3)=1$ if $k \equiv 0 \bmod 4 ;=0$ otherwise;

(c) $d(k, 4)=1$ if $k \equiv 2 \bmod 8 ;=0$ otherwise;

(d) $d(k, 5)=1$ if $k \equiv 6 \bmod 16 ;=0$ otherwise;

(e) $d(k, 6)=2$ if $k \equiv 14 \bmod 16 ;=0$ otherwise;

(f) $d(k, 7)=1$ if $k \equiv 0 \bmod 8 ;=0$ otherwise.

$p=3$ : Here, Mestre has calculated $d(k, \alpha)$ for all $k \leq 136$, and all $\alpha$. The data is again consistent with the above conjecture, and within the range $6 \leq k \leq 136$, one has

(a) $S(k)^{\alpha}=0$ for $\alpha=0,1$ (all $k$ );

(b) $d(k, 2)=1$ if $k \equiv 0 \bmod 6 ;=0$ otherwise;

(c) $d(k, 3)=1$ if $k \equiv 2,4,8$, or $16 \bmod 18 ;=0$ otherwise.

$p=5$ : Mestre's calculations (up to $k=212$ ) suggests that for $k \geq 4$,

(a) $d(k, 1)=1$ if $k \equiv 4,12,16$, and 20 modulo 20 ; and $=0$ otherwise; 
(b) $d(k, 2)=1$ if $k \equiv 2,6$, and 18 modulo 20 , or $k \equiv 28,48,68$, and 88 modulo 100; and $=0$ otherwise.

$p=7$ : Here Mestre's calculations (up to $k=116$ ) suggest that for $k \geq 4$,

$$
d(k, 1)=\left\{\begin{array}{c}
1 \quad \text { if } k \equiv 4,12,16,18,22,24,28,30,34 \\
36,40, \text { and } 42 \text { modulo } 42, \text { and } \\
0 \quad \text { otherwise }
\end{array}\right.
$$

\section{DEFINITION OF $p$-ADIC "FAMILIES" OF EIGENFORMS}

To express our main definition in language that is not very technical, we go at it by successive approximations, describing first a simpler situation, and only then sketching the more complicated general picture.

The main datum, in either case, is a collection of eigenforms of varying weight:

Let $N$ be an integer prime to $p$. To give a $p$-adic family of eigenforms (of tame level $N$ and of slope $\alpha$ ), one must give an eigenform $f_{k}$ of tame level $N$, of slope $\alpha$, and of weight $k$ for every integer $k$ which runs through an arithmetic progression $\mathscr{K}$ of the form

$$
k=k_{0}+t \cdot(p-1) p^{\nu} \quad(t=0,1,2,3, \ldots),
$$

where $k_{0} \geq 1$ and $\nu \geq 0$ are integers.

Kummer families. We shall say that the assignment $k \mapsto f_{k}$ for all $k \in \mathscr{K}$ is a Kummer family if the modular forms $f_{k}$ satisfy the following system of congruences:

If $n$ is an integer $n \geq \nu$, then

$$
f_{k} \equiv f_{k^{\prime}} \quad \text { "modulo } p^{n+1} \text { " }
$$

for any pair $k, k^{\prime} \in \mathscr{K} \operatorname{such}$ that $k \equiv k^{\prime} \bmod (p-1) \cdot p^{n}$.

Here the quotation marks around "modulo $p^{n+1}$ " is simply to remind us that what it means is the following: the difference $f_{k}-f_{k^{\prime}}$, when viewed as power series in $q$, has coefficients in $\mathbb{C}_{p}$ whose ord $p$ 's are greater than or equal to $n+1$.

$R$-families. Let $R$ be a commutative Noetherian algebra over the power series ring in one variable $\mathbb{Z}_{p}[[T]]$, which is finite and free as a module over $\mathbb{Z}_{p}[[T]]$. We shall say that $k \mapsto f_{k}$ is an $R$-family of eigenforms if there is a power series

$$
F=\sum_{n=1} r_{n} q^{n}
$$

with $r_{n} \in R, r_{1}=1$, and if for all $k \in \mathscr{K}$ there are continuous homomorphisms

with $f_{k}=\sum \eta_{k}\left(r_{n}\right) q^{n}$.

$$
\eta_{k}: R \rightarrow \overline{\mathbb{Q}}_{p}
$$

To see the relation between the two definitions, consider the special case of families for which $R=\mathbb{Z}_{p}[[T]]$ and the functions $\eta_{k}$ are given by $T \mapsto$ $(1+p)^{k}-1$. It is easy to see that in this situation the $f_{k}$ will satisfy the 
condition for a Kummer family. It is not clear, however, that any Kummer family must arise in this manner.

\section{EXAmple (Hida's TheORY [5])}

Hida shows that for $p \geq 5$ any eigenform of slope zero, i.e., any eigenform which is $p$-ordinary in the usual terminology (of tame level $N$ ) fits into a $p$ adic family of eigenforms (of tame level $N$, all of slope zero, and where $\nu$, above, can be taken to be 0 ). Hida describes the algebra $R$ in some detail and in particular shows that its rank is equal to the dimension of the slope-zero eigenspace to which the initial form belongs. When this rank is one, he gets a Kummer family.

As a subexample, take the unique cuspidal eigenform $\Delta$ of level $N=1$ and weight 12 , i.e., $\Delta=\eta^{24}=\sum \tau_{n} q^{n}$, where $\eta=q^{1 / 24} \prod\left(1-q^{n}\right)$ is Dedekind's eta function and (by definition) $n \mapsto \tau_{n} \in \mathbb{Z}$ is Ramanujan's tau function. As above, for any prime number $p$, the form $\Delta$ gives rise to two ( $p$-adic) eigenforms $f=\Delta_{p}^{\prime}$ and $f=\Delta_{p}^{\prime \prime}$ whose $U_{p}$-eigenvalues are the two roots of the polynomial $X^{2}-\tau_{p} X+p^{11}$. Then $\alpha\left(\Delta_{p}^{\prime}\right)+\alpha\left(\Delta_{p}^{\prime \prime}\right)=11$, and, ordering these eigenforms so that $\alpha\left(\Delta_{p}^{\prime}\right) \leq \alpha\left(\Delta_{p}^{\prime \prime}\right)$, we have that $\Delta_{p}^{\prime}$ has slope zero exactly when $\tau_{p}$ is not divisible by $p$. This seems to be often the case: for example, the only prime in the range $11 \leq p \leq 65,063$ for which $p$ divides $\tau_{p}$ is $p=2411$. (It would be interesting to know if the same is true for other modular forms, i.e., whether it is true that for any modular form $f$ the primes $p$ such that $a_{p}(f)$ is divisible by $p$ are thinly distributed.)

For any prime number $p$, then, with $\tau_{p}$ nonzero $\bmod p$, there is a $p$-adic family of eigenforms $f_{k}$ of tame level 1 , for $k$ in the arithmetic progression $12+(p-1) \cdot t \quad(t=0,1,2, \ldots)$ with $f_{12}=\Delta_{p}^{\prime}$. The argument above shows that the eigenforms $f_{k}$ actually come from forms of level 1. Can one develop a mechanism to compute (a quantity of) these forms modulo a large power of $p$ ? Even a method for determining the eigenvalues of $U_{p}$ on the $f_{k}$, modulo a large power of $p$, on (a quantity of) these newforms, would be of interest.

\section{A CONJECTURAL GENERALIZATION OF HidA's THEORY}

We believe that there should be a generalization of Hida's theory valid for modular eigenforms of finite slope. The existence of such a theory (already suggested in vague form in [3]) has strong numerical implications, which we formulate below as conjectures. Since we have given two definitions of what we mean by families, we make two conjectures.

Fix $p \geq 5$ and let $f$ be an eigenform of tame level $N$ of weight $k_{0}$ and of any slope $\alpha$. (Recall that $a_{p}(f)$ is never zero, so that $\alpha$ is finite.) Let us refer to $f$ as the initial eigenform.

Conjecture 2. If $\operatorname{dim} S(k)^{\alpha}=1$, there exists a Kummer family $f_{k}$ of tame level $N$, of slope $\alpha$ (and where $\nu$ can be taken to be any integer $\geq \alpha$, the weights $k$ running through the arithmetic progression $k \in \mathscr{K}=\left\{k_{0}+m \cdot p^{\nu}(p-1)\right.$ for $m=0,1,2, \ldots\})$, and such that $f_{k_{0}}$ is equal to the initial eigenform $f$.

Conjecture 3. In general, there is a finite flat $\mathbb{Z}_{p}[[T]]$-algebra $R$, and an $R$ family of eigenforms $f_{k}$ of tame level $N$, of slope $\alpha$ (and where $\nu$ can be taken to be any integer $\geq \alpha$, the weights $k$ running through the arithmetic progression $k \in \mathscr{K}=\left\{k_{0}+m \cdot p^{\nu}(p-1)\right.$ for $\left.\left.m=0,1,2, \ldots\right\}\right)$, and such that $f_{k_{0}}$ is equal 
to the initial eigenform $f$. The rank of the $Z_{p}[[T]]$-algebra $R$ is less than or equal to the dimension of the space $S(k)^{\alpha}$ for any $k \in \mathscr{K}$ with $k>k_{0}$.

One can explore these conjectures in two different ways: theoretically and numerically.

On the theoretical front, see our forthcoming paper [4] whose major goal is to prove a very much weakened form of our three conjectures (continuity of certain characteristic power series).

We became equally fascinated, however, with the prospect of actually numerically checking the conjectures, and computing the plethora of newforms and congruences they predict. This, as we have hinted above, is an interesting project, even in the case covered by Hida's theory, where the conjectures are known to be true. We should note, however, that an important feature of these conjectures is that they predict congruences modulo high powers of the prime number $p$. The existence of collections of eigenforms connected merely by congruences modulo $p$ is guaranteed by a well-known lemma due to Deligne and Serre [2, Lemma 6.11].

\section{ON THE PROLIFERATION OF $p$-ADIC FAMILIES VIA "TWIN" FORMS}

Before going into the numerical evidence in support of the conjectures, we would like to discuss a curious consequence of them.

In this discussion, we will assume we are always in the "Kummer family" situation. Suppose we are given a newform, call it $\varphi$, of level $N$ prime to $p$, and of weight $k_{0}$. As discussed above, we may associate with $\varphi$ and with any prime number $p$ two $p$-adic eigenforms of tame level $N$. These will usually have different slopes $\alpha^{\prime}$ and $\alpha^{\prime \prime}$ satisfying $\alpha^{\prime}+\alpha^{\prime \prime}=k_{0}-1$. But note that, according to the above conjecture, $\varphi_{p}^{\prime}$ fits into a $p$-adic family of eigenforms $f_{k}^{\prime}$, all of slope $\alpha^{\prime}$, while $\varphi_{p}^{\prime \prime}$ fits into a $p$-adic family of eigenforms $f_{k}^{\prime \prime}$, all of slope $\alpha^{\prime \prime}$. Clearly, if $\alpha^{\prime} \neq \alpha^{\prime \prime}$, these two families are not equal.

Note that $\alpha^{\prime}+\alpha^{\prime \prime}=k_{0}-1$, so that for any other weight $k \neq k_{0}, f_{k}^{\prime}$ and $f_{k}^{\prime \prime}$ cannot be a twin pair of $p$-adic eigenforms.

Now for simplicity suppose that $N=1$, and consider this: the argument given above shows that for $k \neq 2 \alpha^{\prime}+2$, the form $f_{k}^{\prime}$ is an "oldform" on $\Gamma_{0}(p)$, and similarly, for $k \neq 2 \alpha^{\prime \prime}+2$, the form $f_{k}^{\prime \prime}$ is old. It follows (with this restriction on $k$ ) that $f_{k}^{\prime}$ and $f_{k}^{\prime \prime}$ each have their own twin companions which imbed (conjecturally) in $p$-adic families of eigenforms of slopes $k-1-\alpha^{\prime}$ and $k-1-\alpha^{\prime \prime}$, respectively. If our conjecture is true, this proliferates!

A particularly interesting "special" case, which formally goes somewhat beyond our conjecture, but may very well follow the format of the conjecture, is the case of the twin form to the noncuspidal form $G_{k}^{*}$ defined in $\S 1$. From the definition of $G_{k}^{*}$ one sees that $G_{k}^{*}=\left(1-p^{k-1} V_{p}\right) G_{k}$. The twin form to $G_{k}^{*}$ is then $\widetilde{G}_{k}:=\left(1-V_{p}\right) G_{k}$, which is a (noncuspidal) eigenform of slope $k-1 .^{3}$ Its Fourier expansion (at the cusp $\infty$ ) has no constant term. Does it imbed in a $p$-adic family, as above, of (cuspidal) eigenforms? We have made a preliminary computation with $k=4$, where we take $p$ to be the (usually forbidden) prime number 3. We find that there exists, for example, a cuspform (as would be conjectured) of weight $58=4+(3-1) 3^{3}$ satisfying the conjectured congruences with $\widetilde{G}_{k}$ modulo $3^{4}$.

\footnotetext{
${ }^{3}$ We call it the evil twin.
} 


\section{Numerical data for Conjecture 2}

Most of our investigations were with the prime numbers $p=5$ and 7. We chose initial eigenforms $f$ of slope $\alpha=1$ and of tame level $N=1$, and of weights $\geq 4$ selected so that $f$ is the unique cuspidal eigenform of its level, slope, and weight. In practice, this restricted us to two situations. For $k=12,16,18,20,22$, and 26 , there is a unique cuspform of level $N=1$, which we could work with when its $p$-slope was equal to 1 . In each of these cases, let $\varphi$ denote this form of level 1 , and let $f^{\prime}$ and $f^{\prime \prime}$ denote the pair of $p$-old twin eigenforms arising from $\varphi$, where $f^{\prime}$ has the smaller slope. The forms $f^{\prime}$ and $\varphi$ are congruent modulo a high power of $p$, so that for our purposes (checking congruences) we could work with either of these two. For $k=4$ and $p=5$ or 7 , there is a unique newform of level $p$, which is of slope 1 .

With these constraints, the $p$-adic initial eigenforms in our computations can be completely identified by their weight $k_{0}$ (and the fact that their tame level is 1 and their slope is 1 ).

$p=5$ : In this case, we would be seeking a Kummer family of $p$-adic eigenforms $f_{k}$ (of tame level 1) of weights $k=k_{0}, k_{0}+20, k_{0}+40$, etc.

Thus the Fourier coefficients of all the $f_{k}$ are congruent to the initial eigenform $f$ modulo 25 and $f_{k} \equiv f_{k+100}$ modulo 125 , etc. We indeed found ${ }^{4}$ such eigenforms of weights $k=k_{0}+20$ and $k_{0}+40$ with initial eigenforms of weights $k_{0}=4,12,16,20$.

Mestre continued these calculations in the case of $k_{0}=12$ to weights $k=$ $12+20 m$ for all integers $m \leq 12$ and found the conjectured eigenforms $f_{k}$ for these weights $k$, whose first 25 Fourier coefficients satisfy the appropriate congruences.

Moreover, he studied all 5-old eigenforms (of tame level 1; Fourier coefficients in $\left.\overline{\mathbb{Q}}_{5}\right)$ of weights $<250$ and whose eigenvalues for $T_{l}(l \neq 5)$ are congruent to those of $f_{k_{0}}$ modulo $5^{2}$, for $k_{0}=12,16$, and 18. (Note: when $k_{0}=18$, the slope of $f_{k_{0}}$ is 2.) All the 5-old eigenforms obtained in Mestre's calculations have Fourier coefficients in $\mathbb{Q}_{5}$, and they are determined by their weights and their slopes. We list them in the three tables below (one table for each of the initial eigenforms $f_{12}, f_{16}$, and $f_{18}$ ), labelling the eigenforms in each weight by their slopes, and with the following further conventions:

(1) We do not list any eigenform of slope $>35$.

Note. This is a harmless simplification of our tables, because the 5-old eigenforms (in our range of weights, of tame level 1 , and such that their $T_{l}$-eigenvalues for $l \neq 5$ are congruent to those of $f_{k_{0}}$ modulo $5^{2}$ ) of slopes $>35$ all happen to be the twins of eigenforms of slope $\leq 35$, and therefore we can reconstruct all the eigenforms (and congruences) from the information given in the tables.

(2) A double line between two listings on our table means that the two corresponding eigenforms are twins.

(3) A single line or, as in the bottom entry in each of the tables, a polygonal arc of shape - connecting two listings means that the two corresponding eigenforms have the property that their $T_{l}$-eigenvalues for $l \neq 5$ are congruent modulo $5^{3}$.

\footnotetext{
${ }^{4}$ By which we mean that we found eigenforms of the correct type whose first 25 Fourier coefficients satisfied the requisite congruences.
} 
TABLE 1

Eigenforms congruent to $f_{12} \quad(=\Delta) \bmod 5^{2}$

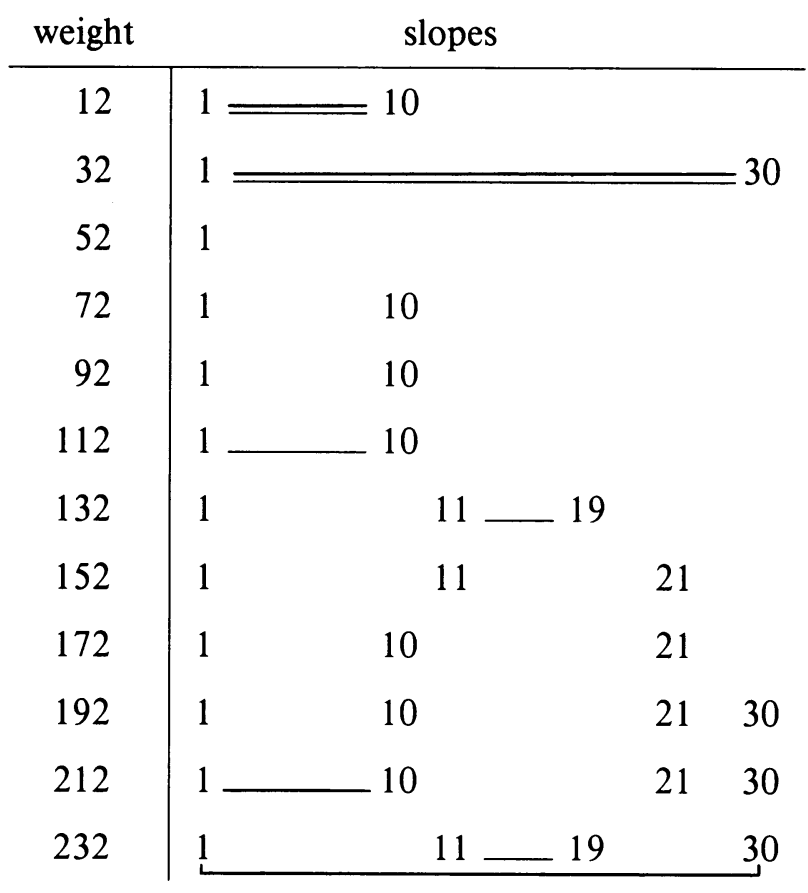

Question. Given two eigenforms $F$ and $G$ appearing in one of the above tables, what is the largest exponent $N \leq \infty$ with the property that their $T_{l}$-eigenvalues for $l \neq 5$ are congruent modulo $5^{N}$ ?

Answer. First, if $F$ and $G$ are twins, their $T_{l}$-eigenvalues for $l \neq 5$ are equal, so one can take $N=\infty$. Second, if there is a single line between $F$ and $G$, then $N=3$. Third, if $F$ and $G$ have the same slopes and their weights differ by 100 , then $N=3$. In general, $N$ is the smallest number $\geq 2$ that it could logically be, from consideration of the tables and the above three assertions.

The three tables came as something of a surprise to us. They are consistent, of course, with our conjectures, but they also display a "regularity" which goes beyond the scope of our conjectures. Even the eigenforms of relatively high slope "behave well" in the arithmetic progression of weights displayed in the tables. One can begin, for example, to see a hint of the phenomenon referred to in $\S 9$ as "proliferation of eigenforms via twin pairs." We also would not have expected that these three tables (related as they are to three completely different initial eigenforms) would have such similar shapes.

$p=7$ : In this case we sought a family of $p$-adic eigenforms $f_{k}$ (of tame level 1 , of slope 1 ) of weights

$$
k=k_{0}, k_{0}+42, k_{0}+84 \text {, etc., }
$$

where the first 25 Fourier coefficients of $f_{k}$ are congruent to the initial eigenform $f$ modulo 49 . Here the weights $k_{0}+84$ were already prohibitively high for us, and so we contented ourselves with the modest project of searching for such forms $f_{k}$ with weight $k=k_{0}+42$. These we found for the initial eigenforms of weights $k_{0}=4,12,16,18$. Mestre extended our calculation, 
TABLE 2

Eigenforms congruent to $f_{16} \bmod 5^{2}$

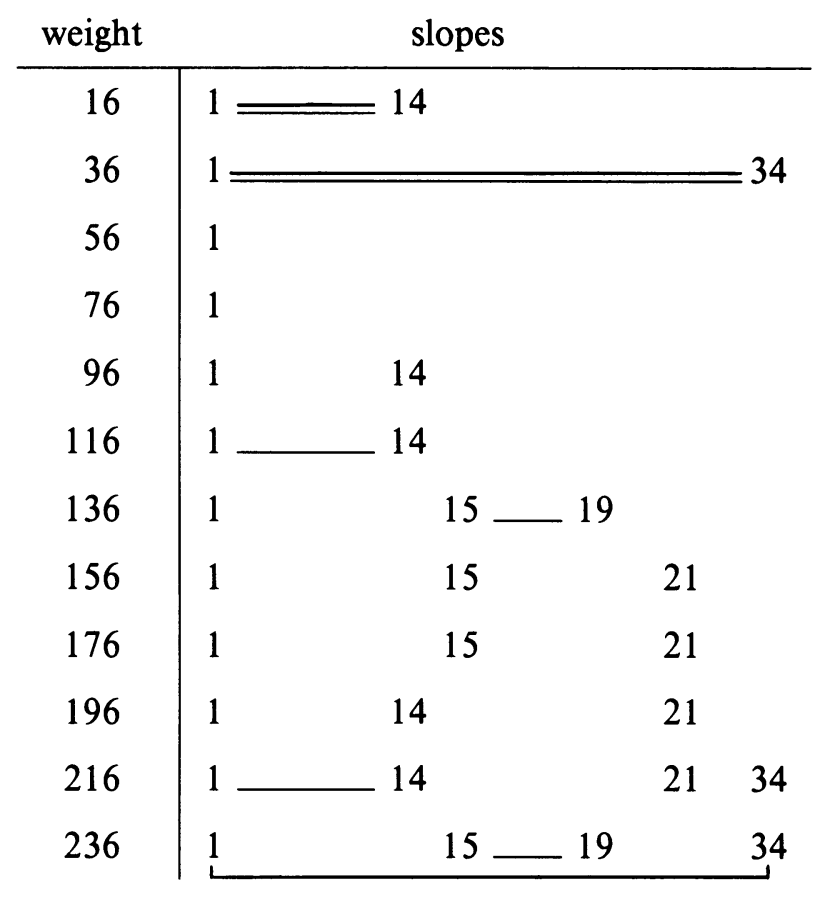

TABLE 3

Eigenforms congruent to $f_{18} \bmod 5^{2}$

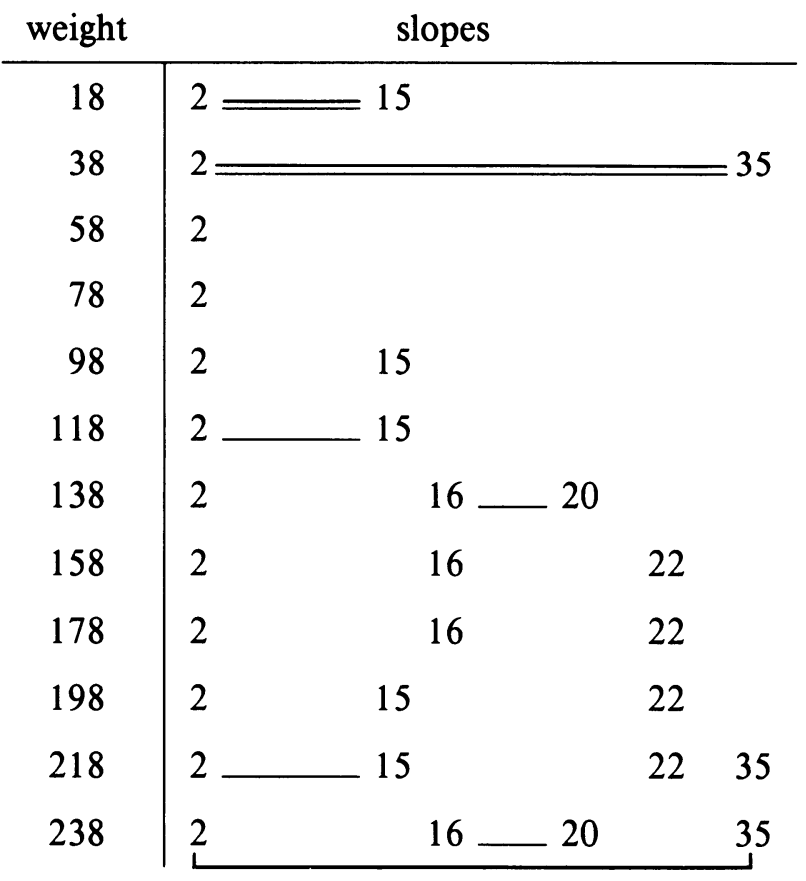


establishing the existence of eigenforms satisfying the appropriate congruences, in the case of $k_{0}=12$, for $k=12+84$ and $12+126$; in the case of $k_{0}=4$, he even found the conjectured congruence modulo $7^{3}$ satisfied by an eigenform of weight $4+6 \cdot 7^{2}$.

Although our theoretical reasons for believing the conjecture cover only the case of prime numbers $p \geq 5$, we felt it worthwhile to search for corresponding families also in the missing cases $p=2$ and 3 (when $p=2$, we interpret " $(p-1) p^{n}$ " as $\left.2^{n-1}\right)$. For these low primes it was feasible to try out some slopes $>1$ as well. For $p=3$ and an initial eigenform of weight $k_{0}=6$ and slope 2 we indeed found the requisite eigenforms of weights 24 and 42 satisfying the required congruence (for their first 25 terms) $\bmod 27$. For $p=2$ we chose an initial eigenform of weight $k_{0}=8$ and slope 3 and found eigenforms of weights 24 and 40 satisfying the appropriate congruences modulo 16.

\section{A TECHNICAL ISSUE}

In making the choices we made to test our conjecture we sought to avoid any instance of our conjecture that has already been proved. Thus, although the computational problem may still be interesting, we avoided initial eigenforms of slope 0 , since they are covered by Hida's theory. There is, however, another situation (in the higher-slope context) where, given an initial eigenform $f$ of weight $k_{0}$, the existence of an eigenform $f_{k}$ with the requisite congruences is guaranteed by a certain lemma of Wiles: If the eigenvalue of the Atkin operator $U_{p}$ on our initial form $f$ occurs with multiplicity one as a root of the characteristic polynomial modulo $p$ of the operator $U_{p}$ on the space of cuspidal modular forms on $\Gamma_{0}(N p)$ of some weight $k$, then Wiles' Lemma [9, Lemma 1.4.3] assures the existence of an $f_{k}$ satisfying the expected congruences (for that $k$ ). In practice, this multiplicity-one phenomenon modulo $p$ occurs only for very low values of $k$ (and there are theoretical reasons as well to expect that it is quite a rare occurrence). In any event, the computations reported above are covered by Wiles' Lemma only in two instances: $k_{0}=4, k=24$ and $k_{0}=12, k=32$, both with $p=5$.

\section{A SAMPLE}

To give a sample of the nature of our original computations, let us take $p=7$ and the initial eigenform $f$ of weight 4 , of tame level 1 , and of slope 1 . Then the Fourier expansion of $f$ is given by

$$
f=q-q^{2}-2 q^{3}-7 q^{4}+16 q^{5}+2 q^{6}-7 q^{7}+15 q^{8}-23 q^{9}-16 q^{10} \ldots
$$

and we are looking for a 7-adic eigenform $f_{46}$ of weight 46 whose Fourier expansion is congruent to the above one modulo 49. This eigenform is one of a "twin pair" of eigenforms coming from a unique cuspidal newform of level 1 and weight 46 , with Fourier coefficients in $\mathbb{Z}_{7}$.

The space of cuspidal modular forms of level 1 and weight 46 is 3-dimensional, and, in what must be the standard straightforward manner, we obtain the characteristic polynomial of the Hecke operator $T_{2}$ on this space:

$$
S^{3}-3814272 S^{2}-44544640241664 S+135250282417024401408 \text {. }
$$

This polynomial is irreducible over $\mathbb{Z}$ and has as roots $\bmod 7$ a single root congruent to 2 and a double root congruent to -1 (the double root splits mod 49 to roots congruent to -1 and 6 ). 
We (with the help of AKCL maxima) can find "the" newform of level 1 and weight 46 "symbolically" in terms of a root $S$ of the above equation. We computed the first 25 terms, the first seven being:

$$
\begin{array}{r}
q \\
+S q^{2} \\
+\left\{\left(S^{2}+29952 S-35184370572864\right) / 336\right\} q^{3} \\
+\left(S^{2}-35184372088832\right) q^{4} \\
+\left\{\left(9495 S^{2}-1930374400 S-334075977367683480\right) / 28\right\} q^{5} \\
-\left\{\left(80088 S^{2}+195005618100 S-2817714217021341696\right) / 7\right\} q^{6} \\
+\left\{\left(389363 S^{2}-15740882088192 S-13756821756459684032\right) / 8\right\} q^{7} \\
+\cdots .
\end{array}
$$

Specializing $S$ to the three roots in $\mathbb{Z}_{7}$ of the characteristic polynomial of $T_{2}$ gives three eigenforms. Specializing $S$ to the root which is $\equiv-99$ modulo 343 gives the 7-adic eigenform $f_{46}$ with the desired congruence modulo 49 (!).

Of course, we could not continue very far in this way (i.e., working over $\mathbb{Z})$, since the integers involved are increasing quite fast. In contrast to our calculations, Mestre's were carried out in the suppler environment of PARI, which in particular allows much of the computation to be done $p$-adically, to any reasonable degree of accuracy.

\section{BIBLIOGRAPHY}

1. A. O. L. Atkin and J. Lehner, Hecke operators on $\Gamma_{0}(m)$, Math. Ann. 185 (1970), 134-160.

2. P. Deligne and J.-P. Serre, Formes modulaires de poids 1, Ann. Sci. École Norm. Sup. 7 (1974), 507-530.

3. F. Gouvêa, Arithmetic of p-adic modular forms, Lecture Notes in Math., vol. 1304, SpringerVerlag, 1988.

4. F. Gouvêa and B. Mazur, Families of p-adic eigenforms (in preparation).

5. $\mathrm{H}$. Hida, Galois representations in $\mathrm{GL}_{2}(\mathrm{Z}[[X]])$ attached to ordinary cusp forms, Invent. Math. 85 (1986), 543-613.

6. N. Jochnowitz, Congruences between systems of eigenvalues of modular forms, Trans. Amer. Math. Soc. 270 (1982), 269-285.

7. N. Katz, p-adic properties of modular schemes and modular forms, Modular Forms in One Variable (W. Kuijk and J.-P. Serre, eds.), Lecture Notes in Math., vol. 350, Springer-Verlag, 1973, pp. 69-190.

8. J.-P. Serre, Formes modulaires et fonctions zêta p-adiques, Modular Functions of One Variable III, Lecture Notes in Math., vol. 350, Springer-Verlag, 1973, pp. 191-268.

9. A. Wiles, On ordinary $\lambda$-adic representations associated to modular forms, Invent. Math. 94 (1988), 529-573.

Department of Mathematics and Statistics, Queens University, Kingston, Ontario K7L 3N6, CANADA

E-mail address: gouvea@heac.mast.QueensU.CA

Current address: Department of Mathematics and Computer Science, Colby College, Waterville, Maine 04901

E-mail address: fqgouvea@colby.edu

Department of Mathematics, Harvard Ujiversity, Cambridge, Massachusetts 02138

E-mail address: mazur@zariski.harvard.edu 